\title{
Game Plan: Communicable Disease Surveillance for Super Bowl XLVIII - New Jersey, 2014
}

\author{
Teresa Hamby*1, Andrew Walsh², Lisa McHugh ${ }^{1}$, Stella Tsai1 and Edward Lifshitz ${ }^{1}$ \\ ${ }^{1}$ New Jersey Department of Health, Trenton, NJ, USA; ${ }^{2}$ Health Monitoring Systems, Inc, Pittsburgh, PA, USA
}

\section{Objective}

To describe the surveillance planning and activities for a largescale event (Super Bowl XLVIII) using New Jersey's syndromic surveillance system (EpiCenter)

\section{Introduction}

In the summer of 2013, the New Jersey Department of Health (NJDOH) began planning for Super Bowl XLVIII to be held on February 2, 2014, in Met Life Stadium, located in the Meadowlands of Bergen County. Surveillance and epidemiology staff in the Communicable Disease Service (CDS) provided expertise in planning for disease surveillance activities leading up to, during, and after the game. A principal component of NJDOH's Super Bowl surveillance activities included the utilization of an existing online syndromic surveillance system, EpiCenter. EpiCenter is a system developed by Health Monitoring Systems, Inc. (HMS) that incorporates statistical management and analytical techniques to process health-related data in real time. As of February, 2014, 75 of New Jersey's 81 acute care and satellite emergency departments (EDs) were connected to this system. CDS staff primarily used EpiCenter to monitor ED visits for unusual activity and disease outbreaks during this event. In addition, $\mathrm{NJDOH}$ and HMS implemented enhanced reports and expanded monitoring of visit complaints.

\section{Methods}

HMS and CDS staff collaboratively established enhanced algorithms to improve statewide ED data monitoring around the game-related activities in addition to the ongoing analyses already included in EpiCenter. New Jersey EDs were requested, via a HAN message, to use "Super Bowl" in their chief complaint fields to flag visits from patients who were involved in game-related activities, and these visits were detected automatically. As well, the implemented enhancements monitored out-of-town registrations, registrations from participating metropolitan areas (Seattle \& Denver), novel word complaints and unlikely complaints. HMS developed a report summarizing these enhanced data points for facilities in proximity to the stadium. These reports were sent to CDS surveillance staff at 6-hour intervals beginning on January 27, 2014, one week prior to the game, and continued until one week after the game (February $\left.10^{\text {th }}\right)$.

\section{Results}

Expected increases in visits to facilities due to the influx of visitors related to the Super Bowl occurred with no anomalies of concern noted. Staff maintained an ongoing comparison of ED visits in 2013 and 2014 to quickly note any dramatic differences (See Figure 1). No differences of concern were noted when accounting for day of week changes from year to year. Twenty ED visits using the key word "Super Bowl" were reported in facilities around the stadium with 17 records coming from one location. In this case, 16 records reflected a misuse of the key word for vomiting cases. The 17th record was a vomiting case related to a Super Bowl event. Continued monitoring showed nothing widespread or sustained in connection to the case. Otherwise, use of the key word was associated with one assault and 2 injuries. The six-hour interval reports provided an important overview to CDS surveillance staff that was summarized in updates to NJDOH's Emergency Command Center. No differences of concern were noted when accounting for day of week changes from year to year.

\section{Conclusions}

Large-scale events pose a challenge to existing surveillance systems where it is expected that additional populations potentially will impact the volume of alerts generated. Implementing enhanced algorithms in an existing system allows better determination between expected anomalies and those that might be of greater concern. The collaboration between NJDOH and HMS, as well as system flexibility within EpiCenter, helps minimize unnecessary response efforts by public health and emergency preparedness personnel during a largescale event such as Super Bowl XLVIII.

Figure 1: EpiCenter Anomalies (Total) - New Jersey

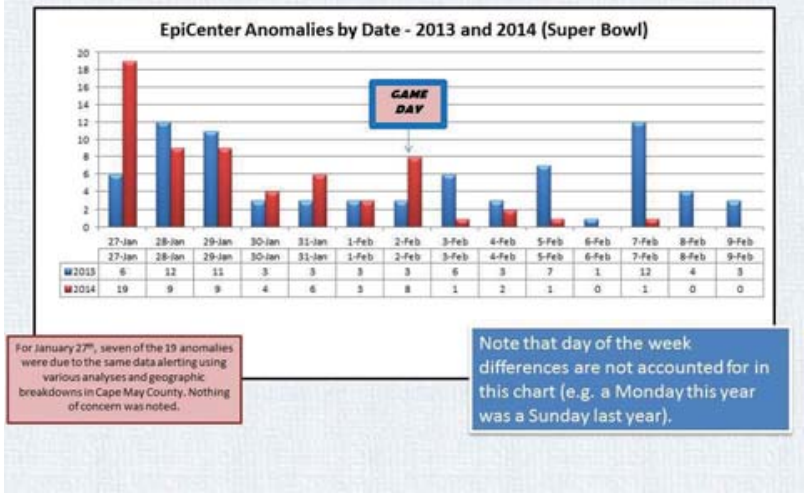

Keywords

Syndromic Surveillance; New Jersey; EpiCenter; Large Scale Event

*Teresa Hamby

E-mail: Teresa.Hamby@doh.state.nj.us 